\title{
Farelo de girassol para aves: composição química e alterações metodológicas na determinação dos valores energéticos e de aminoácidos digestíveis
}

\section{Sunflower meal for broilers: chemical composition and metodologics amendments on determination of energetic values and digestible aminoacids}

\author{
Taciana Maria Moraes de Oliveira ${ }^{1 *}$; Ricardo Vianna Nunes ${ }^{2}$; \\ Simara Márcia Marcato ${ }^{3}$; Eveline Berwanger ${ }^{4}$; Douglas Fernando Bayerle ${ }^{5}$; \\ Rodrigo André Schöne ${ }^{5}$; Rafael Frank ${ }^{5}$; Cleiton Pagliari Sangali ${ }^{1}$
}

\section{Resumo}

O objetivo deste trabalho foi determinar a composição bromatológica e avaliar modificações metodológicas para determinação dos valores energéticos e do perfil de aminoácidos digestíveis do farelo de girassol (FG) para aves. Amostras de FG foram analisadas para a determinação da composição bromatológica. Os valores energéticos foram obtidos pelo método de coleta total de excretas utilizando frangos de corte machos em crescimento, submetidos a cinco dietas contendo 0, 10, 20, 30 e 40\% de substituição de FG em uma ração-referência com quatro repetições. Para determinar os coeficientes de digestibilidade dos aminoácidos e avaliar diferentes métodos de determinação, outro experimento foi conduzido empregando a técnica de alimentação forçada com galos adultos cecectomizados distribuídos em três tratamentos com seis repetições. Os tratamentos consistiram em fornecer $30 \mathrm{~g}$ de FG divididas em duas intubações de $15 \mathrm{~g}$ em intervalos de 12 horas (controle); $30 \mathrm{~g}$ de FG divididas em duas intubações de $15 \mathrm{~g}$ em intervalos de 24 horas e $15 \mathrm{~g}$ de $\mathrm{FG}$ fornecidas em uma única intubação. A composição química determinada foi: 89,51\% MS; 36,46\% PB; 4294 kcal.kg-1 EB; 1,31\% EE; $6,15 \% \mathrm{MM} ; 1,15 \% \mathrm{P} ; 0,33 \% \mathrm{Ca} ; 35,32 \% \mathrm{FDN}$ e $22,30 \%$ FDA. O valor estimado para as variáveis energéticas foi afetado pelo nível de inclusão de FG na ração referência, sendo que o nível de $10 \%$ tende a superestimar os valores, os quais apresentam estabilidade a partir de níveis próximos a $20 \%$. O coeficiente de digestibilidade dos aminoácidos do FG sofre influência da metodologia empregada na sua determinação no que diz respeito ao nível de inclusão para lisina, metionina, treonina, arginina, isoleucina e leucina. Com relação ao período entre a ingestão das doses, não foi verificada diferença entre 12 e 24 horas.

Palavras-chave: Aminoácidos digestíveis, energia metabolizável; valor nutricional

\footnotetext{
${ }^{1}$ Discentes de Doutorado do Programa de Pós-Graduação Stricto Sensu em Zootecnia, Universidade Estadual de Maringá, UEM, Maringá, PR. E-mail: tacideoliveira@hotmail.com, sangalicp@hotmail.com

2 Prof. Dr. do Programa de Pós-Graduação Stricto Sensu em Zootecnia, Universidade Estadual do Oeste do Paraná, UNIOESTE, Marechal Cândido Rondon, PR. E-mail: nunesrv@hotmail.com

${ }^{3}$ Prof $^{a}$ Dr $^{a}$ do Programa de Pós-Graduação Stricto Sensu em Zootecnia, UEM, Maringá, PR. E-mail: smmarcato@uem.br

${ }^{4}$ Discente de Doutorado em Zootecnia, Programa de Pós-graduação Stricto Sensu em Zootecnia, Universidade Federal do Rio Grande do Sul, UFRGS, Porto Alegre, RS. E-mail: evelineberwanger@hotmail.com

5 Discentes de Mestrado do Programa de Pós Graduação Stricto Sensu em Zootecnia, UNIOESTE, Marechal Cândido Rondon, PR. E-mail: douglas_fernandob@hotmail.com; kiko_schone@hotmail.com; rafaelfrankrafael@hotmail.com

* Autor para correspondência
} 


\begin{abstract}
The objective of this study was to determine the bromatological composition and evaluate methodological modifications for determination of sunflower meal (SM) energetic values and digestible aminoacids profile for poultry. Sunflower meal samples were analyzed to determine the bromatological composition. The energetic values were obtained by total feces collection method using growth male broilers, submitted to five diets containing $0,10,20,30$ and $40 \%$ replacement of SM in a reference diet with four replicates. To determine the digestibility coefficients of aminoacids and evaluate diferente determination methods, another experiment was conducted using the technique of forced feeding cecectomized roosters distributed into three treatments with six replicates. The treatments consisted in supplying $30 \mathrm{~g}$ of SM divided into two intubations of $15 \mathrm{~g}$ every 12 hours (control), $30 \mathrm{~g}$ of SM divided into two intubations of $15 \mathrm{~g}$ every 24 hours and $15 \mathrm{~g}$ of SM supplied in a single intubation. The chemical composition determined was: $89.51 \% \mathrm{DM}, 36.46 \% \mathrm{CP}, 4294 \mathrm{kcal} . \mathrm{kg}^{-1} \mathrm{GE}, 1.31 \% \mathrm{CF}, 6.15 \% \mathrm{MM}$, $1.15 \% \mathrm{P}, 0.33 \% \mathrm{Ca} ; 35.32 \% \mathrm{NDF}$ and $22.30 \% \mathrm{ADF}$. The estimated value of the energetic variables was affected by the SM inclusion level in the reference ration, and the $10 \%$ level tends to overestimate the values, which presents stability from near levels of $20 \%$. The SM aminoacids digestibility coefficient is influenced by the methodology used in its determination regarding to the level of inclusion for lysine, methionine, threonine, arginine, leucine and isoleucine. In relation of period between dosis intake, there was no difference between 12 and 24 hours.
\end{abstract}

Key words: Digestible aminoacids, metabolizable energy, nutritional value

\section{Introdução}

O farelo de girassol (FG) resultante da extração do óleo da semente de girassol é considerado uma fonte proteica e pode ser empregado parcialmente como alternativa à utilização do farelo de soja nas rações para frangos de corte. Devido às exigências do mercado consumidor em utilizar outros óleos vegetais além do óleo de soja e o crescente incentivo ao seu cultivo para aproveitamento como biocombustível, verifica-se uma grande disponibilidade de FG, com grande potencial para ser empregado na alimentação animal.

A obtenção do FG baseia-se na extração contínua do óleo utilizando solvente e após este processo, o material segue para tostagem e resfriamento, gerando um produto com valores de proteína bruta ao redor de 28\% (ROSA et al., 2009; TAVERNARI et al., 2010).

Algumas restrições à utilização são apresentadas, tais como o alto nível de fibra (VILLAMIDE; SAN JUAN, 1998) que pode reduzir o aproveitamento de energia (KOCHER et al., 2000; STRINGHINI et al., 2000) sendo necessária a adição de enzimas na ração contendo FG para minimizar os efeitos dos alto teores de polissacarídeos não-amiláceos (PNAs) presentes em sua composição. Observa-se também variação no teor de proteína entre cultivares e a deficiência em lisina quando comparado ao farelo de soja, principalmente em função da quantidade de casca (NRC, 1998).

Diversos trabalhos têm sido conduzidos com o intuito de estabelecer o nível ótimo de inclusão do FG na alimentação de frangos de corte (PINHEIRO et al., 2002; RAMA RAO et al., 2006; ABDELRAHMAN; SALEH, 2007) e de modo geral, a variação nos níveis de inclusão do FG na dieta é atribuída à composição química e valor energético do lote de ingrediente utilizado.

No entanto, um dos maiores entraves à sua maior utilização é a escassez de informações quanto à composição química dos diferentes cultivares, sendo que a determinação do nível energético tem ocupado lugar de destaque, uma vez que a energia regula o consumo de alimento para a maioria das espécies de animais domésticos, gerando a necessidade do estabelecimento de uma relação com os demais nutrientes essenciais (KOLLING; RIBEIRO; KESSLER, 2001). 
Outro ponto a ser considerado para a utilização do FGé a determinação do conteúdo e da digestibilidade dos aminoácidos, permitindo formular dietas mais precisas e econômicas, fazendo com que o animal consuma a quantidade de aminoácidos ajustada à sua exigência nutricional. Assim, os coeficientes de digestibilidade devem ser calculados a fim de se obter o conteúdo aminoacídico que realmente é aproveitado, eliminando o problema de sobras na composição e diminuindo os custos de produção.

Os ensaios de digestibilidade devem ser capazes de fornecer conhecimentos precisos e de maneira rápida. Por este motivo, o desenvolvimento de novas metodologias e o aperfeiçoamento das existentes viabiliza a condução de um maior número de pesquisas e ao mesmo passo, acelera a disposição de novas informações, criando um banco de dados mais consolidado e padronizado acerca da composição química dos alimentos.

O objetivo deste trabalho foi determinar a composição bromatológica e avaliar modificações metodológicas para determinação dos valores energéticos e do perfil de aminoácidos digestíveis do FG para aves.

\section{Material e Métodos}

Os ensaios de metabolismo energético e digestibilidade dos aminoácidos foram realizados no Laboratório de Fisiologia e Metabolismo de Aves e as análises para determinação da composição química do FG foram realizadas no Laboratório de Nutrição Animal, ambos pertencentes à Universidade Estadual do Oeste do Paraná - Unioeste.

\section{Determinação da composição química e dos valores energéticos}

Um ensaio biológico de coleta total de excretas foi realizado com o objetivo de calcular os valores de energia metabolizável aparente (EMA) e energia metabolizável aparente corrigida para o balanço de nitrogênio (EMAn) do FG por meio das equações propostas por Matterson et al. (1965). Após, foram calculados os coeficientes de metabolizabilidade da energia metabolizável aparente (CMA) e da energia metabolizável aparente corrigida para o balanço de nitrogênio (CMAn), com a finalidade de avaliar a eficiência de utilização da energia bruta como energia metabolizável pelas aves.

Este trabalho utilizou 80 frangos de corte machos da linhagem Cobb, de 21 a 31 dias de idade, com peso médio de $913,72 \pm 10,34 \mathrm{~g}$; submetidos a cinco dietas, contendo 0, 10, 20, 30 e 40\% de inclusão de FG em uma ração referência formulada à base de milho e farelo de soja para atender às recomendações propostas por Rostagno et al. (2011), com quatro repetições, totalizando 20 unidades experimentais com quatro aves cada.

O período experimental teve duração de dez dias, sendo cinco dias de adaptação e cinco dias de coleta. Durante todo este período os animais receberam $a d$ libitum água e a ração experimental (Tabela 1). O fotoperíodo empregado foi o de 24 horas.

As coletas foram realizadas duas vezes ao dia, sendo as amostras acondicionadas em sacos plásticos e armazenadas em freezer. Ao final do período de coleta, as excretas seguiram para análises, juntamente com amostras da ração referência (RR) e do FG.

As amostras das excretas foram pré-secas em estufa com circulação forçada de ar a $55^{\circ} \mathrm{C}$ durante 72 horas e assim como as amostras de RR e FG, foram moídas em moinho tipo bola e analisadas de acordo com as técnicas descritas por Silva e Queiroz (2004).

As análises realizadas para RR, FG e excretas foram matéria seca (MS), nitrogênio total (N) e energia bruta (EB). Além dessas análises, o FG também foi submetido à determinação de extrato etéreo (EE), matéria mineral (MM), cálcio ( $\mathrm{Ca})$, fósforo $(\mathrm{P})$, fibra em detergente neutro $(\mathrm{FDN}) \mathrm{e}$ fibra em detergente ácido (FDA). 
Tabela 1. Composição percentual e calculada da ração referência ${ }^{1}$.

\begin{tabular}{|c|c|}
\hline Ingrediente & $\left(\mathrm{g}_{\mathrm{kg}} \mathrm{kg}^{-1}\right)$ \\
\hline Milho grão & 576,81 \\
\hline Farelo de soja & 345,31 \\
\hline Óleo de soja & 45,06 \\
\hline Fosfato bicálcico & 10,98 \\
\hline Calcário & 10,13 \\
\hline Sal comum & 4,57 \\
\hline DL-metionina (99\%) & 2,74 \\
\hline L-lisina $\mathrm{HCl}(78 \%)$ & 1,72 \\
\hline L-treonina & 0,38 \\
\hline Suplemento vitamínico ${ }^{2}$ & 1,00 \\
\hline Suplemento mineral $^{3}$ & 0,50 \\
\hline Cloreto de colina $(60 \%)$ & 0,60 \\
\hline Antioxidante $^{4}$ & 0,20 \\
\hline \multicolumn{2}{|l|}{ Composição calculada } \\
\hline Energia metabolizável $\left(\mathrm{kcal} \mathrm{kg}^{-1}\right)$ & 3150 \\
\hline Proteína bruta $\left(\mathrm{g} \cdot \mathrm{kg}^{-1}\right)$ & 205,00 \\
\hline Cálcio $\left(\mathrm{g} \cdot \mathrm{kg}^{-1}\right)$ & 7,58 \\
\hline Fósforo disponível $\left(\mathrm{g} \cdot \mathrm{kg}^{-1}\right)$ & 3,24 \\
\hline Metionina digestível $\left(\mathrm{g} \cdot \mathrm{kg}^{-1}\right)$ & 5,45 \\
\hline Metinina + Cistina digestível $\left(\mathrm{g} \cdot \mathrm{kg}^{-1}\right)$ & 8,26 \\
\hline Lisina digestível $\left(\mathrm{g} \cdot \mathrm{kg}^{-1}\right)$ & 11,31 \\
\hline Treonina digestível $\left(\mathrm{g} \cdot \mathrm{kg}^{-1}\right)$ & 7,35 \\
\hline Valina digestível (g.kg $\left.{ }^{-1}\right)$ & 8,70 \\
\hline Isoleucina digestível $\left(\mathrm{g} \cdot \mathrm{kg}^{-1}\right)$ & 8,87 \\
\hline Arginina digestível $\left(\mathrm{g} \cdot \mathrm{kg}^{-1}\right)$ & 12,90 \\
\hline Triptofano digestível (g. $\left.\mathrm{kg}^{-1}\right)$ & 2,29 \\
\hline Sódio $\left(\mathrm{g} \cdot \mathrm{kg}^{-1}\right)$ & 2,00 \\
\hline
\end{tabular}

1\% na matéria natural; ${ }^{2}$ Conteúdo: Vit A - 10.000.000 UI; Vit D3 - 2.000.000UI; Vit E - 30.000UI; Vit B1 - 2,0g; Vit B6 4,0g; Ac. Pantotênico - 12,0g; Biotina - 0,10g; Vit K3 - 3,0g; Ac. Fólico - 1,0g; Ac. Nicotílico - 50,0g; Vit B12 - 15.000mcg; Selênio - 0,25g e Veículo q.s.p. - 1.000g; ${ }^{3}$ Conteúdo: $\mathrm{Mg}-$ 16,0g; Fe - 100,0g; Zn - 100,0g; Cu-2,0g; Co-2,0g; I-2,0g e veículo q.s.p. $-1.000 \mathrm{~g} ;{ }^{4} \mathrm{BHT}$

Fonte: Elaboração dos autores.

Ao término do experimento, foram determinadas a quantidade de ração consumida por unidade experimental e a quantidade total de excretas produzidas e utilizando os resultados das análises laboratoriais, foram calculados os valores de EMA, EMAn, CMA e CMAn.

Os dados obtidos para os valores energéticos foram submetidos à análise de variância $(\mathrm{P}<0,05)$ utilizando o Sistema de Análises Estatísticas e Genéticas - SAEG (UFV, 2000).

Determinação dos coeficientes de digestibilidade, dos valores de aminoácidos digestiveis verdadeiros $e$ avaliação de modificações na metodologia empregada

Para determinar os coeficientes de digestibilidade dos aminoácidos e avaliar possíveis alterações no método de determinação dos aminoácidos digestíveis verdadeiros do $\mathrm{FG}$, um experimento foi conduzido utilizando a técnica de alimentação forçada (SIBBALD, 1976) com galos adultos cecectomizados, Leghorn, com peso médio de $1912,10 \pm 133,73 \mathrm{~g}$.

Aotodo, foram utilizados 24 animais, distribuídos em um delineamento inteiramente casualizado, com três tratamentos e seis repetições e um galo por unidade experimental. Concomitantemente, seis animais permaneceram em jejum para determinação das perdas endógenas e metabólicas dos aminoácidos. Os tratamentos consistiram em fornecer $30 \mathrm{~g}$ de $\mathrm{FG}$ divididas em duas intubações de $15 \mathrm{~g}$ em intervalos de 12 horas (controle); $30 \mathrm{~g}$ de FG divididas em duas intubações de $15 \mathrm{~g}$ em intervalos de 24 horas e $15 \mathrm{~g}$ de FG fornecidas em uma única intubação.

Os animais foram alojados em gaiolas individuais e foram submetidos a uma adaptação de cinco dias. Após este período, os animais foram mantidos em jejum por $24 \mathrm{~h}$ e posteriormente, forçados a ingerir o alimento de acordo com o tratamento a que foram submetidos, procedendo-se à coleta total de excretas por 56 h, duas vezes ao dia para evitar fermentação do material. Simultaneamente foi realizada a coleta total de excretas dos animais mantidos em jejum.

O material recolhido foi armazenado em freezer e ao final do período de coleta, o total de excretas foi determinado, posteriormente foram homogeneizadas e seguiram para o Laboratório de Nutrição Animal, aonde foram secas em estufa de 
ventilação forçada por $72 \mathrm{~h}$ a $50^{\circ} \mathrm{C}$. Após a secagem e moagem, amostras das excretas e do FG seguiram para determinação da MS e do $\mathrm{N}$ e depois foram encaminhadas ao laboratório da Evonik Industries $A G$ para determinação do perfil de aminoácidos essenciais por cromatografia líquida de alta performance (HPLC).

Os valores obtidos no tratamento-jejum foram utilizados para realizar as correções correspondentes às perdas metabólicas e endógenas dos aminoácidos. Conhecendo-se as quantidades de aminoácidos ingeridos e excretados, bem como a fração metabólica e endógena obtida com galos em jejum, foram determinados os coeficientes de digestibilidade verdadeira e o conteúdo digestível verdadeiro dos aminoácidos no alimento, por meio da equação proposta por Rostagno e Featherston (1977), para os diferentes tratamentos.

Oprocedimento estatísticoadotado para avaliação das modificações na metodologia empregada para determinação dos aminoácidos digestíveis consistiu em análise de variância $(\mathrm{P}<0,05)$ e posterior comparação dos valores obtidos para o tratamento controle com os dados observados para os demais tratamentos por meio do teste de Dunnett $(\mathrm{P}<0,05)$ utilizando o SAEG (UFV, 2000).

\section{Resultados e Discussão}

A composição química e o valor energético do farelo de girassol estão intimamente associados às condições de cultivo (tais como solo, clima e cultivar) e processamento do alimento, as quais não são uniformes. Esta falta de padronização não pode ser totalmente controlada, pois alguns fatores como perfil químico do solo de cultivo e condições climáticas não são passíveis de alteração. No entanto, quando se trata do processamento, a aplicação de normativas que visam estipular uma metodologia para obtenção do produto contribui para que este se apresente com menor variabilidade na composição.
No caso do FG empregado neste estudo, este foi obtido da extração de óleo por meio de solvente químico (hexano) e após seguiu para tostagem e resfriamento. Neste processo, existem muitas variáveis, tais como o teor de nutrientes da semente de girassol, o método de extração do óleo (que pode ser químico, mecânico ou ambos), o solvente empregado (hexano ou éter etílico), tempo e temperatura de tostagem, entre outros. Assim, a padronização destas possíveis fontes de variação levaria à obtenção de um produto com qualidade nutricional conhecida e consolidada.

Levic, Sredanovic e Duragic (2005) relatam que em geral, o teor de proteína bruta do FG utilizado na Europa varia entre 33 e $37 \%$, faixa na qual se encaixa o teor descrito por este trabalho (Tabela 2). Diversos estudos têm reportado valores de proteína bruta dentro da variação descrita por Levic, Sredanovic e Duragic (2005), tais como San Juan e Villamide (2001) com 33,14\%; Ravindran et al. (2005) que relatam teor de 35,20\% e Rezaei e Hafezian (2007) que observaram níveis de 35,94\%.

Tabela 2. Composição proximal e valor de energia bruta do farelo de girassol ${ }^{1}$.

\begin{tabular}{lc}
\hline Composição & \\
\hline Matéria seca (\%) & 89,51 \\
Proteína bruta (\%) & 36,46 \\
Energia bruta (kcal.kg-1) & 4294 \\
Extrato etéreo (\%) & 1,31 \\
Matéria mineral (\%) & 6,15 \\
Fósforo (\%) & 1,15 \\
Cálcio (\%) & 0,33 \\
Fibra em detergente neutro (\%) & 35,32 \\
Fibra em detergente ácido (\%) & 22,30 \\
\hline
\end{tabular}

${ }^{1}$ Valores expressos na matéria natural

Fonte: Elaboração dos autores.

No entanto, a maioria dos autores descrevem valores inferiores, como Kocher et al. (2000), Sauvant, Perez e Tran (2004); Tavernari et al. (2010) e Hamedi, Rezaian e Shomali (2011) que 
observaram respectivamente, valores de 26,03; 27,$70 ; 28,09 ; 25,60 \%$ de proteína bruta no FG.

Esta grande variabilidade quanto aos teores de proteína devem-se principalmente ao elevado conteúdo de fibra presente na composição do FG, o que é dependente da maior ou menor presença de casca no coproduto, estando diretamente relacionado com a forma de processamento.

Rosa et al. (2009) ao avaliarem dois cultivares de girassol e dois métodos de extração do óleo (semente inteira e semente sem casca) relataram que os teores de proteína foram maiores quando o FG não continha casca durante sua obtenção. Segundo estes autores, o FG obtido a partir de sementes com casca apresentou respectivamente, 14,73 e 18,22\% de proteína e quando a semente foi previamente descascada, os teores de proteína foram de 17,65 e $19,66 \%$.

O valor de energia bruta foi inferior aos 4633 kcal.kg-1 relatados por San Juan e Villamide (2001). Outros estudos como os de Silva et al. (2012) e Mantovani et al. (2000) apresentam teores semelhantes sendo, respectivamente: 4324 e 4306 kcal. $\mathrm{kg}^{-1}$.

Assim como para o conteúdo de proteína, o nível de energia bruta do FG também depende do seu processamento, sendo inversamente proporcional à quantidade de casca presente e diretamente proporcional ao conteúdo de óleo residual. Este fato pode ser evidenciado nos dados apresentados por Villamide e San Juan (1998) que relataram valores que variaram de $4710 \mathrm{kcal} . \mathrm{kg}^{-1}$ de energia bruta para amostras com 3,61\% de extrato etéreo a $4557 \mathrm{kcal}$. $\mathrm{kg}^{-1}$ para amostras contendo $0,73 \%$.

Desta forma, o baixo nível de extrato etéreo encontrado pode ter influenciado no valor de energia bruta do alimento. Em geral, o conteúdo de extrato etéreo apresentado foi inferior ao descrito por Mantovani et al. (2000) e Freitas et al. (2004) que analisando amostras de FG observaram valores de 1,40 e 1,34 respectivamente.

Os valores de fibra foram inferiores aos observados por Stringhini et al. (2000) que expuseram valores de $42,15 \%$ para FDN e $31,68 \%$ para FDA e Carellos et al. (2005) que observaram teores de $43,57 \%$ de FDN e $32,96 \%$ de FDA. Assim sendo, a alta concentração de fibra no FG pode ser considerada um fator limitante ao seu uso nas rações de aves, podendo exercer efeito sobre o aproveitamento da energia. Nunes et al. (2008) determinando os coeficientes de metabolizabilidade da energia bruta de diferentes alimentos para aves, observaram em seu estudo que os alimentos com alto teor de fibra apresentaram menores coeficientes de metabolizabilidade, comprovando a baixa digestibilidade da fibra no trato gastrointestinal das aves.

$\mathrm{O}$ teor de matéria mineral apresentado pelo $\mathrm{FG}$ foi superior aos dados descritos por Rodrigues et al. (2004) com 4,41\%; Selvaraj e Purushothaman (2004) com 3,50\% e Silva et al. (2012) que observaram nível de 5,35\%.

Com relação aos níveis de cálcio, o valor foi superior aos $0,22 \%$ observados por Carellos et al. (2005) e 0,19\% relatados por Rama Rao et al. (2006). No entanto, Silva et al. (2012) relataram nível semelhante $(0,34 \%)$. Além disso, Mantovani et al. (2000) e Tavernari et al. (2010) descrevem teores superiores $(0,45 \%$ e $0,83 \%$, respectivamente).

A maioria da literatura consultada apresenta níveis inferiores de fósforo quando comparado ao determinado por este trabalho, tais como Mantovani et al. (2000) que relataram 1,13\%; Rodrigues et al. (2004) $0,67 \%$ e Araújo et al. (2011) $1,00 \%$.

Com relação aos valores de EMA, EMAn, CMA e CMAn, a análise de variância demonstrou que as variáveis foram afetadas pelos tratamentos aplicados $(\mathrm{P}<0,05)$ e a análise de regressão $(\mathrm{P}<0,05)$ indicou o modelo quadrático como o que melhor se ajustou aos dados (Tabela 3 ). 
Tabela 3. Valores de energia metabolizável aparente (EMA), energia metabolizável aparente corrigida pelo balanço de nitrogênio (EMAn), coeficiente de metabolizabilidade da energia aparente (CMA) e coeficiente de metabolizabilidade da energia aparente corrigida pelo balanço de nitrogênio (CMAn) para os diferentes níveis de inclusão de farelo de girassol ${ }^{1}$.

\begin{tabular}{lcccc}
\hline \multirow{2}{*}{ Variáveis } & \multicolumn{4}{c}{ Nível de inclusão do farelo de girassol (\%) } \\
\cline { 2 - 5 } & 10 & 20 & 30 & 40 \\
\hline EMA (kcal.kg-1) & $2030,79 \pm 114,82$ & $1418,04 \pm 118,14$ & $1435,86 \pm 91,86$ & $1459,00 \pm 134,41$ \\
EMAn (kcal.kg-1) & $1839,19 \pm 154,28$ & $1315,51 \pm 112,70$ & $1318,28 \pm 96,52$ & $1298,67 \pm 130,23$ \\
CMA (\%) & $47,29 \pm 2,67$ & $33,02 \pm 2,75$ & $33,44 \pm 2,14$ & $33,99 \pm 3,13$ \\
CMAn (\%) & $42,83 \pm 3,59$ & $30,64 \pm 2,62$ & $30,70 \pm 2,25$ & $30,24 \pm 3,03$ \\
\hline
\end{tabular}

${ }^{1}$ Expressos na matéria natural.

Fonte: Elaboração dos autores.

A partir do nível de 10\%, ocorreu uma diminuição nos valores estimados de EMA e EMAn e estabilização dos mesmos a partir de 19,24\% de inclusão para EMA ( $\mathrm{Y}=4007,9-267,1 \mathrm{X}+6,9408 \mathrm{X}^{2}$, $\left.\mathrm{R}^{2}=0,99\right)$; com um nível de 1438,22 kcal. $\mathrm{kg}^{-1}$ e $21,29 \%$ para EMAn $\left(\mathrm{Y}=3194,0-177,0 \mathrm{X}+4,1558 \mathrm{X}^{2}\right.$, $\mathrm{R}^{2}=0,99$ ); com um nível de $1309,34 \mathrm{kcal} \cdot \mathrm{kg}^{-1}$.

Os dados referentes aos coeficientes de metabolizabilidade da energia do FG, assim como para os valores energéticos, apresentaram níveis mais elevados para $10 \%$ de inclusão do alimento teste, havendo estabilização da curva a partir de $19,23 \%$ de inclusão para CMA (Y=93,3942$6,2301 \mathrm{X}+0,162 \mathrm{X}^{2}, \mathrm{R}^{2}=0,99$ ); apresentando CMA de $33,49 \%$ e $21,33 \%$ de inclusão para CMAn $\left(\mathrm{Y}=74,2818-4,1083 \mathrm{X}+0,0963 \mathrm{X}^{2}, \mathrm{R}^{2}=0,99\right)$; sendo esta igual a $30,45 \%$.

A estimativa da EMA do alimento é diretamente afetada pelo nível de substituição da ração referência pelo alimento teste. Segundo Sakomura e Rostagno (2007) para alimentos com alto teor de fibra, como no caso do FG, recomenda-se substituir $20 \%$ da ração referência para determinar a EMA pelo método de coleta total de excretas.

A substituição acima dos teores de inclusão utilizados normalmente nas rações de produção pode, de acordo com Penz Junior, Kessler e Brugalli (1999), subestimar os valores de energia dos alimentos em ensaios de metabolismo. Partindo desta premissa, pode-se assegurar que o emprego de níveis de inclusão inferiores aos recomendados superestima os valores de energia, como pode ser evidenciado neste estudo, onde, tanto a EMA como a EMAn apresentaram valores superiores para $10 \%$ de inclusão quando comparado aos outros níveis de inclusão, nos quais os valores energéticos apresentaram-se semelhantes.

No caso do FG, grande parte dos autores utiliza o nível de $20 \%$ de substituição para estimar seu conteúdo energético. Deste modo, os valores de EMA e de EMAn encontrados foram, respectivamente, inferiores aos 2141 e 1983 kcal. kg-1 apresentados por Tavernari et al. (2010), que incluíram 20\% de FG em uma ração referência, do mesmo modo que os coeficientes de metabolizabilidade também foram inferiores (48,34\% para CMA e 44,77\% para CMAn), assim como Silva et al. (2012) que obtiveram 1781 kcal.kg-1 para e EMA e $1607 \mathrm{kcal}$. $\mathrm{kg}^{-1}$ para EMAn, apresentando CMA de $41,18 \%$ e CMAn de $37,16 \%$.

Com o mesmo percentual de inclusão, Hamedi, Rezaian e Shomali (2011) expuseram para EMA níveis de $1753 \mathrm{kcal} . \mathrm{kg}^{-1}$, também sendo superiores ao observado neste estudo. No entanto, Mushtaq et al. (2009) ao adicionarem 20 e 30\% de FG em uma ração basal, encontraram, respectivamente, 1126 e 1132 kcal. $\mathrm{kg}^{-1}$ para EMA, valores inferiores aos relatados neste trabalho. 
Ao comparar os níveis de 20 e $40 \%$ de substituição da dieta referência por FG, Freitas et al. (2004) verificaram que a substituição em 40\% proporcionou redução em relação ao nível de $20 \%$ para os valores de EMA (de 2216 para $1966 \mathrm{kcal}$. $\mathrm{kg}^{-1}$ ), EMAn (de 1902 para 1711 kcal.kg'), CMA (de $48,19 \%$ para $42,75 \%$ ) e CMAn (de 41,36 para $37,21 \%$ ). Os autores atribuem essa redução ao alto teor de fibra do alimento, que reduz a digestibilidade dos nutrientes em virtude do aumento da taxa de passagem, dificultando o acesso das enzimas digestivas aos nutrientes durante a digestão.

Para todas as variáveis, as maiores estimativas foram obtidas com o nível de inclusão de $10 \%$. Este comportamento dos dados vem corroborar com o fato de que a inclusão de $10 \%$ do alimento teste em ensaios de metabolismo de energia superestima os resultados, uma vez que próximo do nível preconizado $(20 \%)$, a curva tende a se estabilizar.

Grande parte das variações na metabolizabilidade da energia das dietas relacionam-se aos componentes fibrosos, como a lignina e os PNAs. Além disso,
Nunes et al. (2005) citam que o conteúdo de proteína bruta, ácidos graxos e minerais também são fatores que contribuem para as diferenças nos valores energéticos dos alimentos.

Neste estudo, o nível de extrato etéreo observado foi inferior ao citado por diversos trabalhos. Isto indica uma presença menor de lipídios disponíveis para os processos oxidativos, que após a lipólise geram glicerol e ácidos graxos, resultando na liberação de energia no organismo das aves ocorrida no fígado (quando na forma de glicerol) e na mitocôndria das células de outros tecidos (quando ácidos graxos), o que se torna mais significativo ao considerar o fato de que os lipídios correspondem aos nutrientes com maior aporte energético.

Além dos valores energéticos, o conteúdo de aminoácidos (Tabela 4) também se apresenta como fator determinante na escolha pela utilização ou não de um alimento alternativo. Para isso, são necessários ensaios de digestibilidade para que os níveis de aminoácidos possam ser utilizados de maneira mais segura na formulação de ração.

Tabela 4. Valores dos coeficientes de digestibilidade verdadeira dos aminoácidos essenciais, aminoácidos totais (AAt) e digestíveis verdadeiros (AAd).

\begin{tabular}{lccc}
\hline Aminoácido & Coeficiente de digestibilidade (\%) & $\mathrm{AAt}^{1}$ & $\mathrm{AAd}$ \\
\hline Lisina & $82,10 \pm 2,31$ & 1,970 & 1,618 \\
Metionina & $88,89 \pm 1,57$ & 0,837 & 0,744 \\
Metionina + Cistina & $84,38 \pm 2,22$ & 1,819 & 1,535 \\
Treonina & $78,02 \pm 4,27$ & 1,659 & 1,457 \\
Arginina & $92,11 \pm 1,35$ & 2,566 & 2,289 \\
Histidina & $87,78 \pm 2,70$ & 1,173 & 1,030 \\
Valina & $79,77 \pm 3,31$ & 1,992 & 1,589 \\
Isoleucina & $82,55 \pm 2,87$ & 1,588 & 1,311 \\
Leucina & $85,69 \pm 2,57$ & 2,902 & 2,486 \\
Fenilalanina & $84,84 \pm 2,60$ & 1,672 & 1,418 \\
\hline
\end{tabular}

${ }^{1}$ Valores expressos na matéria natural.

Fonte: Elaboração dos autores.

Entre as metodologias empregadas, a permitirdeterminaras perdas endógenas metabólicas ingestão forçada de alimento com galos adultos e fazer uso de animais mais resistentes ao processo cecectomizados é uma das mais utilizadas por de cecectomia e à metodologia em si. No entanto, 
modificações que visam aprimorar a metodologia são sempre desejadas, desde que facilitem a obtenção dos resultados, gerando economia de tempo e causando menos desconforto às aves.

De modo geral, o FG apesar de possuir uma proteína relativamente rica em aminoácidos sulfurados, apresenta, para as rações de frangos de corte, uma deficiência em lisina, quando comparado ao farelo de soja. No presente estudo, empregando análise por meio de HPLC para aminoácidos totais e o método tradicional de determinação de aminoácidos digestíveis, os níveis de lisina total e digestível foram superiores aos relatados por diversos autores (SAN JUAN; VILLAMIDE, 2001; DHAWALE, 2005; TAVERNARI et al., 2010).

Ravindran et al. (1999a), ao comparar os métodos de coleta total e coleta ileal de excretas na determinação da digestibilidade dos aminoácidos de vários ingredientes utilizando frangos de corte com 35 dias, observaram para o $\mathrm{FG}$, níveis de $1,19 \%$ para lisina total e 0,82 e $0,78 \%$ para lisina digestível pelos métodos de coleta ileal e coleta total, respectivamente. Também pelo método de coleta ileal, Stringhini et al. (2000) observaram níveis de $0,80 \%$ de lisina para o farelo de girassol.

As tabelas da Fundación Española de Desarrollo de la Nutrición Animal (FEDNA) e do Institut National de la Recherche Agronomique (INRA) da França apresentam valores de aminoácidos digestíveis obtidos por meio da coleta ileal. Segundo estas tabelas, os níveis de lisina digestível variam entre 0,91 e 1,07\% (DE BLAS BEORLEGUI; MATEOS; REBOLAR, 2003) e entre 0,83 e 0,98\% (SAUVANT; PEREZ; TRAN, 2004). As tabelas brasileiras de Rostagno et al. (2011) utilizam dados obtidos tanto com galos cecectomizados quanto com coleta ileal e apresentam um valor médio de $0,78 \%$ de lisina digestível para o FG.

Apesar do teor de lisina do FG ser relativamente baixo, este alimento pode ser utilizado como fonte de proteína na dieta, mas desde que em substituição parcial ao farelo de soja. Mushtaq et al. (2006) estudando a inclusão de complexo multienzimático em dietas com dois níveis de FG e três níveis de lisina digestível verificaram que a conversão alimentar das aves melhorou linearmente com o nível de inclusão da lisina.

$\mathrm{O}$ fato de o $\mathrm{FG}$ ser rico em aminoácidos sulfurados equilibra com a sua deficiência em lisina, pois a suplementação de metionina também é um fator a ser considerado na escolha da matriz nutricional das dietas, principalmente ao ponderar o fato de que a metionina é o primeiro aminoácido limitante para frangos de corte.

Do mesmo modo que para lisina, os níveis de metionina e metionina+cistina foram superiores aos observados na literatura. Stringhini et al. (2000) relataram níveis de $0,53 \%$ para metionina digestível e $0,80 \%$ para metionina+cistina digestível, as tabelas de De Blas Beorlegui; Mateos e Rebolar (2003) especificam um conteúdo que varia de 0,65 a $0,74 \%$ de metionina e 1,12 a $1,22 \%$ de metionina + cistina e as tabelas francesas de Sauvant, Perez e Tran (2004) expõem uma variação entre 0,59 e $0,71 \%$ para metionina e 0,99 e $1,19 \%$ para metionina+cistina.

Para os demais aminoácidos, também foi constatado que os níveis descritos por este estudo, tanto para aminoácidos totais como para digestíveis, são superiores aos expostos por Ravindran et al. (1999b), Stringhini et al. (2000), San Juan e Villamide (2001), De Blas Beorlegui; Mateos e Rebolar (2003), Sauvant, Perez e Tran (2004), Levic, Sredanovic e Duragic (2005) e Tavernari et al. (2010).

Ao trabalhar com alimentos alternativos, intensificam-se as variações observadas nos coeficientes de digestibilidade dos aminoácidos, havendo grande importância da digestibilidade desses nutrientes, quando da formulação de rações, para que se possa obter máximo desempenho.

Os valores de digestibilidade para os aminoácidos essenciais do FG reportados por Lemme, Ravindran e Bryden (2004) em seu trabalho sobre 
a digestibilidade ileal de aminoácidos em alimentos para frangos de corte foram superiores aos citados neste estudo.

Nouri-Emamzadeh e Yaghobfar (2009) ao conduzirem experimento visando determinar a influência da cecectomia na digestibilidade dos aminoácidos dos farelos de soja, canola e girassol, utilizando galos inteiros e cecectomizados, também apresentaram coeficientes de digestibilidade superiores, com exceção da metionina e da fenilalanina para os galos cecectomizados.

San Juan e Villamide (2001) e Tavernari et al. (2010) apresentaram coeficientes de digestibilidade da lisina menores $(67,67$ e $81,16 \%$, respectivamente) do que este experimento. O mesmo foi observado para a metionina, sendo que os autores relataram coeficientes de $86,51 \%$ e $88,71 \%$, respectivamente.

A arginina foi o aminoácido que apresentou o maior coeficiente de digestibilidade, sendo superior aos de Villamide e San Juan (1998), que observaram coeficientes digestibilidade de 90,22; 91,94 e 92\% para FG com 32, 35 e 37\% de proteína bruta, respectivamente e ao coeficiente de 91,61\% reportado por Tavernari et al. (2010).

O menor coeficiente de digestibilidade foi obtido para a treonina e juntamente com a valina, isoleucina e leucina foram inferiores à todas as fontes pesquisadas (VILLAMIDE; SAN JUAN, 1998; SAN JUAN; VILLAMIDE, 2001; LEMME; RAVINDRAN; BRYDEN, 2004; NOURI-EMAMZADEH; YAGHOBFAR, 2009; TAVERNARI et al., 2010).

Muitos são os fatores que afetam a digestibilidade da proteína e dos aminoácidos dos alimentos, como as características ligadas a planta, seja agronômica ou ao processamento, as características ligadas ao animal como peso, sexo, idade, status fisiológico e genótipo, e os procedimentos experimentais como a metodologia empregada na determinação da digestibilidade e do nível de consumo (NOBLET; LE GOFF, 2001).
De acordo com Leeson e Summers (2001), o processamento térmico também pode gerar produtos resistentes à hidrólise enzimática, os quais são provenientes da reação de Maillard que tende a ocorrer quando o processamento térmico é excessivo, sendo que a lisina é o aminoácido mais afetado usualmente pelo superaquecimento devido sua maior susceptibilidade à esta reação (PARSONS, 1996).

A metodologia empregada nos ensaios metabólicos também pode influenciar a digestibilidade dos aminoácidos. Neste estudo, o coeficiente de digestibilidade verdadeira dos aminoácidos foi maior ( $\mathrm{P}<0,05)$ para o nível de consumo de $15 \mathrm{~g}$ do alimento para lisina, metionina, treonina, arginina, isoleucina e leucina. No entanto, o intervalo entre a ingestão do alimento não afetou o coeficiente de digestibilidade dos aminoácidos $(\mathrm{P}>0,05)$, sendo igual tanto para intervalos de ingestão de 12 horas, quanto para o de 24 horas (Tabela 5).

Através deste resultado e considerando-se o fato de que alimentos com alto teor de fibra aumentam a taxa de passagem, é correto afirmar que quanto mais alimento fibroso o animal ingerir, maior será a taxa de passagem. No caso deste trabalho, a taxa de passagem tendeu a ser menor em animais que ingeriram apenas $15 \mathrm{~g}$ de $\mathrm{FG}$ do que aqueles que ingeriram $30 \mathrm{~g}$, logo, os coeficientes de digestibilidade serão maiores, pois o alimento teve mais tempo de exposição ao epitélio intestinal.

Em contrapartida, Borges et al. (2005) ao avaliarem os coeficientes de digestibilidade dos aminoácidos utilizando a técnica da alimentação forçada com dois níveis de consumo (25 e 50 g), observaram que nos níveis de consumo menores os valores de digestibilidade aparente foram significativamente menores. Estes autores afirmam que a ingestão de pequena quantidade de alimento poderia induzir um catabolismo corporal aumentando a porção de aminoácidos endógenos das aves, resultando em valores subestimados dos aminoácidos digestíveis dos alimentos. 
Tabela 5. Coeficiente de digestibilidade verdadeira dos aminoácidos essenciais segundo o intervalo entre as ingestões e o nível de consumo do farelo de girassol ${ }^{1 .}$.

\begin{tabular}{lcccc}
\hline \multirow{2}{*}{ Aminoácido } & \multicolumn{2}{c}{ Coeficiente de digestibilidade verdadeira (\%) } & \multirow{2}{*}{ CV (\%) } \\
\cline { 2 - 4 } & $30 \mathrm{~g} / 12 \mathrm{~h}^{2}$ & $30 \mathrm{~g} / 24 \mathrm{~h}$ & $15 \mathrm{~g}$ & 7,20 \\
Lisina & $82,10 \pm 2,31$ & $80,88 \pm 3,83$ & $89,86 \pm 9,50^{*}$ & 4,27 \\
Metionina & $88,89 \pm 1,57$ & $86,34 \pm 3,21$ & $95,61 \pm 5,63^{*}$ & 6,70 \\
Metionina + Cistina & $84,38 \pm 2,22$ & $81,32 \pm 4,68$ & $92,56 \pm 8,53^{*}$ & 16,40 \\
Treonina & $78,02 \pm 4,27$ & $75,51 \pm 7,29$ & $87,65 \pm 21,22$ & 3,03 \\
Arginina & $92,11 \pm 1,35$ & $91,09 \pm 2,33$ & $96,49 \pm 4,09^{*}$ & 7,77 \\
Histidina & $87,78 \pm 2,70$ & $83,12 \pm 3,19$ & $91,76 \pm 11,01$ & 10,17 \\
Valina & $79,77 \pm 3,31$ & $78,61 \pm 4,88$ & $88,43 \pm 13,23$ & 8,27 \\
Isoleucina & $82,55 \pm 2,87$ & $79,84 \pm 4,45$ & $90,41 \pm 10,84^{*}$ & 6,87 \\
Leucina & $85,69 \pm 2,57$ & $82,97 \pm 3,82$ & $92,18 \pm 9,25^{*}$ & 7,28 \\
Fenilalanina & $84,84 \pm 2,60$ & $83,07 \pm 4,06$ & $91,52 \pm 9,82$ & \\
\hline Média & $84,28 \pm 7,01$ & $82,02 \pm 5,77$ & $91,75 \pm 10,43$ & \\
\hline
\end{tabular}

${ }^{1}$ Na matéria natural; ${ }^{2}$ Tratamento controle; *Diferem estatisticamente do tratamento controle pelo teste Dunnett $(\mathrm{P}<0,05)$.

Fonte: Elaboração dos autores.

\section{Conclusões}

O valor estimado para as variáveis energéticas foi afetado pelo nível de inclusão de farelo de girassol na ração referência e o coeficiente de digestibilidade dos aminoácidos do alimento sofreu influência da metodologia empregada na sua determinação.

\section{Agradecimentos}

Ao apoio financeiro cedido pela Fundação Araucária de Apoio à Pesquisa do Estado do Paraná.

Todos os ensaios biológicos foram autorizados pelo Comitê de Ética na Experimentação Animal e Aulas Práticas da Universidade Estadual do Oeste do Paraná sob o protocolo n 04411/2011.

\section{Referências}

ABDElRAHMAN, M. M.; SALEH, F. H. Performance of broiler chickens fed on corn-sunflower meal diets with ß-glucanase enzyme. Jordan Journal of Agricultural Sciences, Amman, v. 3, n. 3, p. 272-280, 2007.

ARAÚJO, L. F.; ARAÚJO, C. S. S.; PETROLI, N. B.; LAURENTIZ, A. C.; ALBUQUERQE, R.; TRINDADE NETO, M. A. Sunflower meal for broilers of 22 to 42 days of age. Revista Brasileira de Zootecnia, Viçosa, MG, v. 40, n. 10, p. 2142-2146, 2011.
BORGES, F. M. O.; ROSTAGNO, H. S.; SAAD, C. E. P.; LARA, L. B.; TEIXEIRA, E. A. Efeito do nível de ingestão sobre a digestibilidade dos aminoácidos em frangos de corte. Ciência Agrotécnica, Lavras, v. 29, n. 2, p. 444-452, 2005.

CARELlOS, D. C.; LIMA, J. A. F.; FIALHO, E. T.; REITAS, R. T. F.; SILVA, H. O.; BRANCO, P. A. C.; SOUZA, Z. A.; VIEIRA NETO, J. Evaluation of sunflower meal on growth and carcass traits of finishing pigs. Ciencia Agrotécnica, Lavras, v. 29, n. 1, p. 208-215, 2005.

DHAWALE, A. Peanut and sunflower meal to replace soybean meal. World Poultry, Hanzestraat, v. 21, n. 2, p. 12-13, 2005.

FREITAS, E. R.; SAKOMURA, N. K.; NEME, R.; SANTOS, F. R. Determinação da digestibilidade dos nutrientes e da energia metabolizável da semente e do farelo de girassol para frangos de corte. In: REUNIÃO ANUAL DA SOCIEDADE BRASILEIRA DE ZOOTECNIA, 41., 2004, Campo Grande. Anais... Campo Grande: Sociedade Brasileira de Zootecnia, 2004. p. 1-4.

DE BlAS BEORLEGUi, C.; MATEOS, G. G.; REBOLLAR, P. G. Tablas FEDNA de composición y valor nutritivo de alimentos para la fabricación de piensos compuestos. 2. ed. Madri: Fundación Española para el Desarrollo de la Nutrición Animal, 2003. 253 p.

HAMEDI, S.; REZAIAN, M.; SHOMALI, T. Histological changes of small intestinal mucosa of cocks due to sunflower meal single feeding. American Journal of Animal and Veterinary Sciences, New York, v. 6, n. 4, p. 171-175, 2011. 
KOCHER, A.; CHOCT, M.; PORTER, M. D.; BROZ, J. The effects of enzyme addition to broiler diets containing high concentrations of canola or sunflower meal. Poultry Science, Savoy, v. 79, n. 12, p. 1767-1774, 2000.

KOLLING, A. V.; RIBEIRO, A. M. L.; KESSLER, A. M. Efeito de diferentes relações de energia e proteína e de alimentação por livre escolha sobre o desempenho e composição corporal de frango de corte. In: REUNIÃO ANUAL DA SOCIEDADE BRASILEIRA DE ZOOTECNIA, 38., 2001, Piracicaba. Anais... Piracicaba: Sociedade Brasileira de Zootecnia, 2001. p. 1-4.

LEESON; S.; SUMMERS, J. D. Scott's nutrition of the chicken. 4. ed. Guelph: University Books, 2001. 591 p.

LEMME, A.; RAVINDRAN, V.; BRYDEN, W. L. Ileal digestibility of amino acids in feed ingredients for broilers. World's Poultry Science Journal, Cambridge, v. 60, n. 4, p. 421-435, 2004.

LEVIC, J. D.; SREDANOVIC, S. A.; DURAGIC, O. M. Sunflower meal protein as a feed for broilers. APTEFF, v. 36, n. 1, p. 3-10, 2005.

MANTOVANI, C.; FURLAN, A. C.; MURAKAMI, A. E.; MOREIRA, I.; SCAPINELLO, C.; SANTOLIN, M. R. da R. Chemical composition and energetic values of sunflower meal and seed for broiler chickens. Acta Scientiarum, Maringá, v. 22, n. 3, p. 745-749, 2000.

MATTERSON, L. D.; POTTER, L. M.; STUTZ, M. W.; SINGSEN, E. P. The metabolizable energy of feed ingredients for chikens. Storrs: Connecticut University, Agricultural Experiment Station, 1965. 11 p. (Research report).

MUSHTAQ, T.; SARWAR, M.; AHMAD, G.; NISA, M. U.; JAMIL, A. Influence of sunflower meal based diets supplemented with exogenous enzyme and digestible lysine on performance, digestibility and carcass response of broiler chickens. Animal Feed Science and Technology, Amsterdam, v. 149, n. 3-4, p. 275-286, 2009.

The influence of exogenous multienzyme preparation and graded levels of digestible lysine in sunflower meal-based diets on the performance of young broiler chicks two weeks post hatching. Poultry Science, Savoy, v. 85, n. 12, p. 2180-2185, 2006.

NATIONAL RESEARCH COUNCIL - NRC. Nutrient requirements of swine. 10. ed. Washington, D.C.: National Academy of Sciences, 1998. 189 p.

NOBLET, J.; LE GOFF, G. Effect of dietary fiber on the energy value of feeds for pigs. Animal Feed Science and Technology, Amsterdam, v. 90, n. 1, p. 35-52, 2001.

NOURI-EMAMZADEH, A.; YAGHOBFAR, A. Influence of caecectomy on digestibility of amino acids for soybean, canola and sunflower meals in adult cockerels. The Journal of Poultry Science, Tsukuba, v. 46, n. 1, p. 19-24, 2009.

NUNES, R. V.; POZZA, P. C.; NUNES, C. G. V.; CAMPESTRINI, E.; KÜHL, R.; ROCHA, L. D.; COSTA, F. G. P. Valores energéticos de subprodutos de origem animal para aves. Revista Brasileira de Zootecnia, Viçosa, MG, v. 34, n. 4, p. 217-224, 2005.

NUNES, R. V.; ROSTAGNO, H. S.; GOMES, P. C. NUNES, C. G. V.; POZZA, P. C.; ARAUJO, M. S. Coeficientes de metabolizabilidade da energia bruta de diferentes ingredientes para frangos de corte. Revista Brasileira de Zootecnia, Viçosa, MG, v. 37, n. 1, p. 8994, 2008.

PARSONS, C. M. Digestible amino acids for poultry and swine. Animal Feed Science Technology, Amsterdam, v. 59, n. 1, p. 147-153, 1996.

PENZ JUNIOR, A. M.; KESSLER, A. M.; BRUGALLI, I. Novos conceitos de energia para aves. In: SIMPÓSIO INTERNACIONAL SOBRE NUTRIÇÃO DE AVES, 1999, Campinas. Anais... Campinas: Fundação Apinco de Ciência e Tecnologia Avícolas, 1999. p. 1-24.

PINHEIRO, J. W.; FONSECA, N. A. N.; SILVA, C. A.; CABRERA, L.; BRUNELI, F. A.; TAKAHASHI, S. E. Farelo de girassol na alimentação de frangos de corte em diferentes fases de desenvolvimento. Revista Brasileira de Zootecnia, Viçosa, MG, v. 31, n. 3, p. 1418-1425, 2002. Suplemento.

RAMA RAO, S. V.; RAJU, M. V. L. N.; PANDA, A. K.; REDDY, M. R. Sunflower seed meal as a substitute for soybean meal in commercial broiler chicken diets. British Poultry Science, Edinburgh, v. 47, n. 5, p. 592-598, 2006.

RAVINDRAN, V.; CABAHUG, S.; RAVINDRAN, G.; BRYDEN, W. L. Influence of microbial phytase on apparent ileal amino acid digestibility of feedstuffs for broilers. Poultry Science, Savoy, v. 78, n. 5, p. 677-706, 1999b.

RAVINDRAN, V.; HEW, L. I.; RAVINDRAN, G.; BRYDEN, W. L. A comparison of ileal digesta and excreta analysis for the determination of amino acid digestibility in food ingredients for poultry. British Poultry Science, Edinburgh, v. 40, n. 2, p. 266-274, 1999a.

Apparent ileal digestibility of amino acids in feed ingredients for broiler chickens. Animal Science, Liverpool, v. 81, n. 1, p. 85-97, 2005.

REZAEI, M.; HAFEZIAN, H. Use of different levels of high fiber sunflower meal in commercial Leghorn type layer diets. International Journal of Poultry Science, Faisalabad, v. 6, n. 6, p. 431-433, 2007. 
RODRIGUES, K. F.; NAGATA, A. K.; RODRIGUES, P. B.; FREITAS, R. T. F.; LEITE, R. S. Inclusão do farelo de girassol em dietas de frangos de corte suplementadas com $\beta$-glucanase. In: REUNIÃO ANUAL DA SOCIEDADE BRASILEIRA DE ZOOTECNIA, 41., 2004, Campo Grande. Anais... Campo Grande: Sociedade Brasileira de Zootecnia, 2004. p. 1-5.

ROSA, P. M.; ANTONIASSI, R.; FREITAS, S. C.; BIZZO, H. R.; ZANOTTO, D. L.; OLIVEIRA, M. F.; CASTIGLIONI, V. B. R. Chemical composition of brazilian sunflower varieties. Helia, Novi Sad, v. 32, n. 50, p. 145-156, 2009.

ROSTAGNO, H. S.; ALBINO, L. F. T.; DONZELE, J. L.; GOMES, P. Z.; OLIVEIRA, R. F.; LOPES, D. C.; FERREIRA, A. S.; BARRETO, S. L. T.; EUCLIDES, R. F. Tabelas brasileiras para aves e suínos: composição de alimentos e exigências nutricionais. 3. ed. Viçosa: UFV, Departamento de Zootecnia, 2011. 252 p.

ROSTAGNO, H. S.; FEATHERSTON, W. R. Estudos de métodos para determinar disponibilidade de aminoácidos em pintos. Revista Brasileira de Zootecnia, Viçosa, MG, v. 6, n. 1, p. 64-76, 1977.

SAKOMURA, N. K.; ROSTAGNO, H. S. Métodos de pesquisa em nutrição de monogástricos. Jaboticabal: FUNEP, 2007. 283 p.

SAN JUAN, L.; VILLAMIDE, M. Nutritional evaluation of sunflower products for poultry as affected by the oil extraction process. Poultry Science, Savoy, v. 80, n. 4, p. 431-437, 2001.

SAUVANT, D.; PEREZ, J. M.; TRAN, G. Tablas de composición y de valor nutritivo de las materias primas destinadas a los animales de interés ganadero: cerdos, aves, bovinos, ovinos, caprinos, conejos, caballos, peces. Madrid: Mundi-Prensa, 2004. 195 p.
SELVARAJ, R. K.; PURUSHOTHAMAN, M. R. Nutritive value of full-fat sunflower seeds in broiler diets. Poultry Science, Savoy, v. 83, n. 3, p. 441-446, 2004.

SIBBALD, I. R. A bioassay for true metabolizable energy in feedstuffs. Poultry Science, Savoy, v. 55, n. 1, p. 303308, 1976.

SILVA, D. J.; QUEIROZ, A. C. D. Análises de alimentos: métodos químicos e biológicos. 3. ed. Viçosa: UFV, 2004. $235 \mathrm{p}$.

SILVA, E. A.; ALBINO, L. F. T.; ROSTAGNO, H. S.; RIBEIRO JUNIOR, V.; VIEIRA, R. A.; CAMPOS, A. M. A.; MESSIAS, R. K. G. Chemical composition and metabolizable energy values of feedstuffs for broiler chickens. Revista Brasileira de Zootecnia, Viçosa, v. 41, n. 3, p. 648-654, 2012.

STRINGHINI, J. H.; CAFÉ, M. B.; FERNANDES, C. M.; ANDRADE, M. L.; ROCHA, P. T.; LEANDRO, N. S. M. Avaliação do valor nutritivo do farelo de girassol para aves. Ciência Animal Brasileira, Goiânia, v. 1, n. 2, p. 123-126, 2000.

TAVERNARI, F. C.; MORATA, R. L.; RIBEIRO JÚNIOR, V.; ALBINO, L. F. T.; DUTRA JUNIOR, W. M.; ROSTAGNO, H. S. Avaliação nutricional e energética do farelo de girassol para aves. Arquivo Brasileiro de Medicina Veterinária e Zootecnia, Belo Horizonte, v. 62, n. 1, p. 172-177, 2010.

UNIVERSIDADE FEDERAL DE VIÇOSA - UFV. Sistema de análises estatísticas e genéticas - SAEG. Versão 8.0.Viçosa, 2000. 142 p.

VILLAMIDE, M. J.; SAN JUAN, L. D. Effect of chemical composition of sunflower seed meal on its true metabolizable energy and amino acid digestibility. Poultry Science, Savoy, v. 77, n. 12, p. 1884-1892, 1998. 
